\title{
Intuitive and Efficient Control of Real-Time MRI Scan Plane Using a Six-Degree-of-Freedom Hardware Plane Navigator
}

\author{
Dingrong Yi, Jeff Stainsby, and Graham Wright \\ Sunnybrook \& Women's College Health Sciences Center, Imaging Research, \\ and University of Toronto, Department of Medical Biophysics, 2075 Bayview Avenue, \\ Toronto, Ontario, M4N 3M5, Canada \\ \{dingryi, stainsby, gawright\}@sten.sunnybrook.utoronto.ca
}

\begin{abstract}
For applications of real-time MRI, it is important but difficult to efficiently prescribe the next scan plane and obtain visual feedback on the prescription. This work addresses these issues with a 6-degreeof-freedom Plane Navigator. The Plane Navigator is a mechanical arm with integrated input and output functionality while being statically balanced. In the input mode, by holding and moving the surface normal of the physical representation of the scan plane, the operator can intuitively place the scan plane at a position with any orientation within a few milliseconds. In the output mode, the Plane Navigator automatically places the physical representation of the scan plane to reflect its position and orientation (pose)[PR1] relative to a patient domain with a maximum delay of half a second. Application examples in MRI cardiac imaging are also described.
\end{abstract}

\section{Introduction}

Magnetic resonance imaging (MRI) has long been used to visualize anatomical structures in human patients. The development of new techniques for the rapid acquisition of MR images during the past few years has made it possible to achieve interactive acquisitions at 10-20 frames per second with sub-millimeter resolution [9]. However, one of the major obstacles that prevent real-time MRI from expanding its clinical application is the lack of absolute pointing hardware tools for interactive and intuitive manipulation of the scan plane. The high updating frequency of 10 to 20 frames per second is only feasible at one single location of a scan plane or with automatically prescribed scan planes. Without a suitable hardware device, it is difficult to interactively change the scan plane location quickly enough to fully take advantage of such imaging efficiency.

Most of the current work on interface design focuses on software development to provide graphical tools [1, 3], 6]. Well-designed graphical user interfaces written in software can provide some of the required prescription flexibility by providing a 2D projection of the oblique cut plane corresponding to the current scan plane in the context of a pre-acquired imaging study [6]. However the visual feedback of the location and orientation of the current scan plane provided 
is limited by the flat screen. Meanwhile without a suitable hardware tool it is difficult to precisely control the scan plane in a coordinated manner.

Existing 6-DOF hardware devices such as Spaceball, SpaceMouse and Flock of Birds have been used to improve the efficiency of scan plane prescription [4, [2]. However, none of these device can visually represent a static scan plane position. In this work, we demonstrate how the 6-DOF Plane Navigator can be used to provide intuitive and interactive control of scan plane during real-time MRI.

\section{Method}

\subsection{The Plane Navigator}

The Plane Navigator is designed to integrate the following functions in order to provide an intuitive and interactive control of the scan plane: (1) 6-DOF manual inputs, (2) automatic display of a 6-DOF spatial location, and (3) static balance which enables the display of both manual and automatic operations and reduces the required force to be exerted on the stylus for motion during manual operations.

The Plane Navigator is a mechanical arm consisting of a series of mechanical linkages that support a planar surface, referred to as the proxy plane (Figure 1a). A stylus is fixed and perpendicular to the surface to represent its normal. The linkages are connected through 6 rotational joints $O_{i}(\mathrm{i}=1,2, \ldots, 6)$. The Plane Navigator has a spherical workspace of radius $R=2 *\left|\mathrm{O}_{2} \mathrm{O}_{3}\right|=2 *\left|\mathrm{O}_{3} \mathrm{O}_{6}\right|$, centered at $\mathrm{O}_{2}$. The second joint $\mathrm{O}_{2}$ is also the origin $\mathrm{O}_{w}$ of world coordinate system. The combined effects of rotating along the A-A, B-B and C-C axes determines the center position $O_{6}$ of the proxy plane. The rotation along the D$\mathrm{D}, \mathrm{E}-\mathrm{E}$ and F-F axes further determines the orientation of the proxy plane. The decoupling of positioning from orientation is realized by setting the cross-point of the axes D-D, E-E and F-F to be the center $O_{6}$ of the proxy plane. At each joint an optical encoder is coupled with a motor. Static balance is realized by symmetric design, light weight materials, friction, stabilizing motor torque, and where applicable counter-weight blocks.

The prototyped Plane Navigator is constructed using aluminum alloy, inexpensive components such as 300 count optical encoders E4 (US Digital) and custom modified Hitech servos (Tower Hobby). A single PCI MultiQ I/O board (Quanser) is used to read 6 encoders. The motors are controlled through 6 of the 8 data pins of the parallel port of a personal computer, with real-time linux to precisely control the required timing. Encoder readings are used as feedback for position control. The radius of the spherical work space is $220 \mathrm{~mm}$, which allows a 1:1 ratio between the armature and MR scanner dimension for real-time cardiac imaging (Figure 1 $\mathrm{b}$ ). The positioning accuracy for manual mode is $0.8 \mathrm{~mm}$ and for automatic mode is $1.8 \mathrm{~mm}$.[PR2] In automatic mode, the accuracies of rotation along axes D-D, E-E and F-F are 1.5, 0.6, and 0.6 degrees respectively (Figure 17). There is a cylindrical inaccessible zone along the $O_{w} Z_{w}$ axis with radius of $43 \mathrm{~mm}$ due to mechanical occlusion (Figure 1a). 


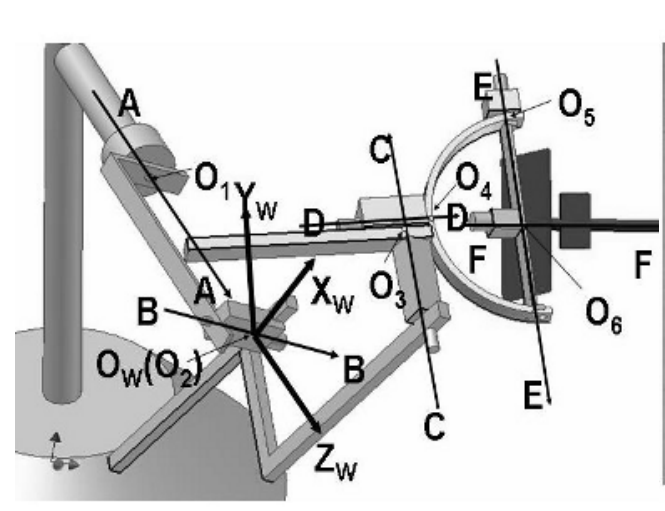

(a)

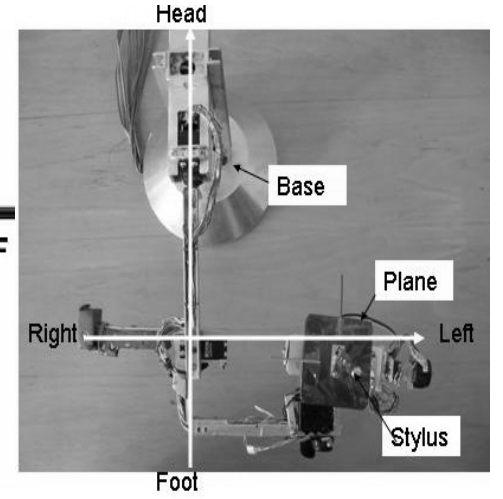

(b)

Fig. 1. (a) Illustration of the design of the Plane Navigator. A-A through F-F are the rotational axes, $O_{i}(\mathrm{i}=1,2, \ldots, 6)$ are the rotational joints, and $O_{w} X_{w} Y_{w} Z_{w}$ is the fixed world coordinate system used to describe the workspace of the Plane Navigator. (b) Illustrates one way of configuring the workspace of the Plane Navigator to correspond to the coronal view of cardiac MR imaging of a human subject in supine position.

\subsection{Registering the Plane Navigator with a MRI Scanner}

To demonstrate how the Plane Navigator can be used for real-time MRI, let us consider its application to cardiac imaging, which is one of the major applications of real-time MRI. For imaging the heart, only a portion of the workspace of the Plane Navigator is needed. Therefore the space occupied by the heart in the patient domain is registered with and centered at $(0.4,0.4,0)$ of the normalized workspace of the Plane Navigator. Figure 1b illustrates such a configuration of the workspace of the Plane Navigator to match a patient in supine position. The world coordinate system $O_{w} X_{w} Y_{w} Z_{w}$ of the Plane Navigator matches the scanner's coordinate system in a $1: 1$ ratio. The axis $Z_{w}$ is the first linkage. The physical representations of the center $O_{w}$ and the axis $Z_{w}$ of the world coordinate system will not change their positions significantly, regardless of the current location and orientation of the proxy plane. Therefore they can serve as the spatial references. For example the physical representation of the $Z_{w}$ axis can well represent the backbone of a supine patient (Figure 1b).

\section{Results}

The Plane Navigator is utilized as an input device for MRI scan-plane prescription by having the user hold and maneuver the surface normal (stylus) of the proxy plane, and thus reach an arbitrary position and orientation. Meanwhile, operations involving only rotations at a fixed position are easy to perform. The computer inserts the encoder readings into the forward kinematics to calculate 
the position and orientation of the proxy plane. This information is constructed into a $4 \mathrm{x} 4$ matrix to be sent to the scanner. In such a way the user can simultaneously enter coordinated $\mathrm{x}, \mathrm{y}, \mathrm{z}$ positions as well as pitch, yaw and roll rotations to control the scan plane. The time required to sample the encoders and reconstruct the $4 \times 4$ geometric matrix is less than one millisecond.

When the Plane Navigator is activated to take the role of output device the translation vector and $3 \times 3$ rotation matrix of a given prescription is converted to the appropriate joint angles using inverse kinematics. These joint angles are then sent to the motors to automatically translate and rotate the proxy plane to physically reflect the position and orientation of the scan plane. The maximum delay in output mode is $500 \mathrm{~ms}$, which corresponds to moving the proxy plane from one edge to the extreme opposite edge of the workspace.

\subsection{Interactive Control of Real-Time MRI Scan Plane}

The Plane Navigator has been tested to interactively control and automatically represent the scan plane of a $1.5 \mathrm{~T}$ scanner $(\mathrm{GE} \mathrm{CV} / \mathrm{i})$. Currently it connects to a personal computer running real-time Linux. A second computer running a realtime MRI interface has direct control of the scanner. The latter sends geometry information such as translation and rotation to the scanner, displays the reconstructed image. etc. The two computers communicate through a socket-based server process to transport the $4 \mathrm{x} 4$ geometric matrix containing the position and orientation of the scan plane [8].
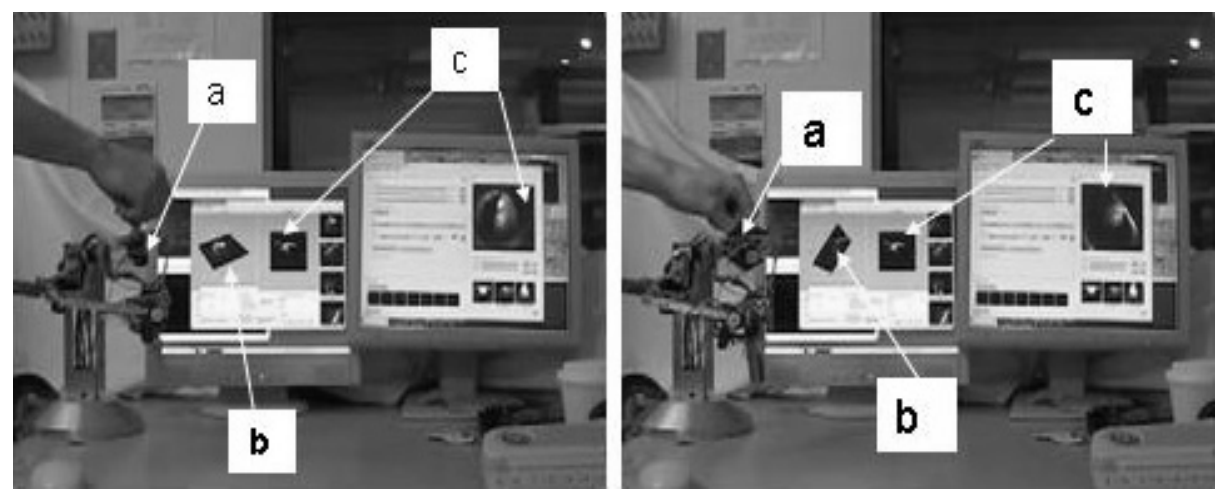

Fig. 2. Two snapshots to indicate how an operator interactively controls the real-time MRI scan plane (b) using the Plane Navigator (a). Image (c) is the resulting real-time MR image of a healthy human subject. The scan plane (b) is graphically displayed in the context of a pre-acquired imaging study of the same human subject [6].

The Plane Navigator provides interactive control of the real-time MRI scan plane in its input mode (Figure 2). The imaging parameters are: GRE sequence with minimum TE, TR=50 milliseconds, Flip angle=30 degree, field of view is 
$20 \mathrm{~cm}$. Display rate of 15 frames per second was achieved using sliding window reconstruction technique. The update rate of the $4 \times 4$ geometric matrix obtained from the Plane Navigator was $200 \mathrm{~Hz}$. However, due to the delay of the socket-based communication between the Plane Navigator and the computer which has direct access to the MRI scanner, currently there is a noticeable delay between the user manipulation of the proxy plane and the appearance of the corresponding image (indicated in Figure 2 as a and $\mathbf{c}$ respectively). This delay is approximately $200 \mathrm{~ms}$. The delay is anticipated to be reduced to be a few milliseconds longer than the time required for imaging acquisition and reconstruction, which is currently around $70 \mathrm{~ms}$. One way to do this is to let all the processes run within a single computer using shared memory technique or to use simple first-in-first-off buffers for the transportation of the shared geometry matrix. [PR3]

The Plane Navigator's position could be initialized to preset locations, to facilitate subsequent manual fine adjustments to accommodate variability among different subjects. Alternatively the user can use sliders as indicated in [8] to prescribe the next scan plane by translating and rotating the current one. The Plane Navigator can automatically track and follow the prescription. This automatic tracking provides direct visual feedback on the effects of the software-controlled spatial operation. In addition the visibility of the physical representation of the scan plane and its $\mathrm{x}, \mathrm{y}$ and $\mathrm{z}$ axis guides the operator's determination of which direction the current scan plane should move in order to achieve the imaging goal.

\subsection{Intuitive Control of the Scan Plane}

The Plane Navigator aids the user's intuition in several ways. There is a direct mapping between the workspace of the device and the scanner workspace. Indeed, both spaces can be described by similar coordinate systems. The Plane Navigator provides a physical representation of the current scan plane in a workspace that has a one-to-one correspondence to the subject in the scanner. The capability of holding its pose in a physically visible, fixed reference coordinate system provides the operator with direct visual feedback of the spatial location of the scan plane under manipulation. Such physical correspondence and visual feedback aids comprehension of the location and orientation of the current scan plane relative to patient anatomy.

Holding and manipulating the stylus for 3D inputs is as natural as holding a pen. Delicate[PR4] static balance at each of the joints enables the user to freely manipulate the stylus. The user is not required to expend effort and become fatigued by countering gravity and carrying the device during operation. In addition it is convenient to halt and later resume an unfinished operation.

Cardiologists without training on the Navigator can intuitively manipulate the proxy plane of the device to obtain various standard views of a human heart. Figure 3 illustrates how a cardiologist would place the proxy plane to match standard cardiac views. 
(1)
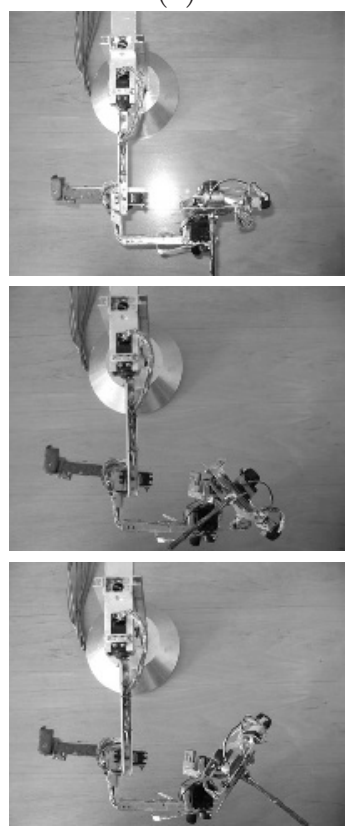

$(2)$
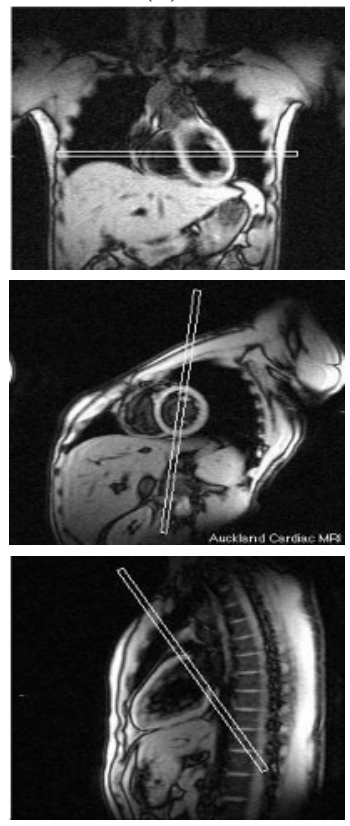

$(3)$
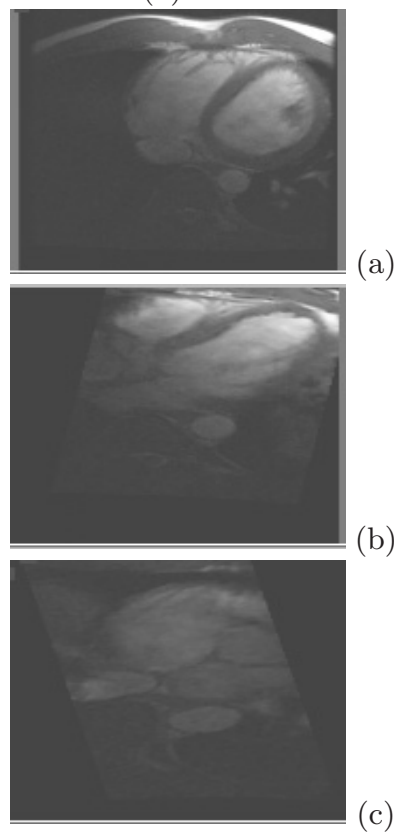

Fig. 3. Illustrating the correspondence between the placements of the proxy plane of the Plane Navigator and the standard cardiac views. Column (1) shows the actual placements of the proxy plane by a cardiologist to match the locations in the patient domain as shown in column (2) to obtain standard cardiac views as shown in column (3). Row (a), (b) and (c) roughly correspond to the axial, long axis and short axis views of a healthy human heart. Column (2) images were borrowed with permission from the anatomy tutorial provided at SCMR official website (http://www.scmr.org/education/atlas/intro).

\section{Discussion}

There are several advantages of the Plane Navigator over software-based scan plane control tools and other 6-DOF hardware input tools. is that The Navigator can simultaneously provide coordinated scan plane manipulation in the required 6-DOF space in an intuitive and interactive manner within a few milliseconds[PR5]. This capability may eliminate the current bottleneck restricting imaging efficiency in real-time MRI. Meanwhile it presents the user direct visual feedback on the location and orientation of the resulting scan plane in a patient domain. Its capability of automatic tracking of the scan plane enhances visualization when navigating with a software tool. The physical representation of the scan plane and its attached image coordinate system help the user comprehend the anatomy contained in the real-time image in the patient domain. 
With the Plane Navigator an operator can interactively navigate the scan plane, guided directly by the individual anatomy and can track the resulting MR images at a display rate of 10 to 20 frames per second. In other words, it is feasible to manually track and visualize moving and deforming anatomical structures regardless of complex dynamic processes. This will certainly improve the diagnostic power of MRI.[PR7] Though designed for the maneuver of realtime MRI scan planes, it can also be used to interactively control the view plane in $3 \mathrm{D}$ visualization (Figure 3 ).

A disadvantage of the Plane Navigator is that the operation space of the proxy plane is constrained within its spherical workspace, hence it is not as flexible as the cable tethered devices nor the wireless handheld components such as the Flock of Birds (Ascension Technology Corporation) 1 . All absolute pointing mechanical armatures such as MicroScribe by Immersion Corp, Freedom6S designed by Hayward [5] and commercialized by MPB2, Phantom by Sensable 3 and the Plane Navigator suffer this inconvenience due to their fixed-size physical workspaces.

A unique feature of the Plane Navigator is its ability to maintain static balance, whereas other pointing devices will drift in their position and orientation. Preliminary evaluation with cardiologists and real-time MRI researchers indicates that static balance facilitates resuming an unfinished operation. Formal human performance experiments are under preparation to quantitatively evaluate the value of static balance, output functionality and their combination for an absolute-pointing device. Freedom6S to some extent has similar static balance capability. However due to the requirement of high fidelity force output, its stylus may drift away from the position where the user released it. Another difference between the Plane Navigator and haptic devices is that the latter use motors to generate forces instead of precise positions. The most closely related device that the authors are aware of is the slave robot of da Vinci Surgical System designed by Salisbury [7] and commercialized by Intuitive Surgica [4. The operator can control both manually and automatically the tip position of the surgical tool holder of that robot. Despite this similarity, the Plane Navigator is far more affordable.[PR9]

\section{Conclusion}

A 6-DOF absolute pointing hardware tool was developed for interactive realtime MRI scanning to enhance the efficiency and intuitiveness of scan plane localization. This system could be applied wherever fast, intuitive localization or tracing in $3 \mathrm{D}$ is required.

\footnotetext{
${ }^{1}$ http://www.ascension-tech.com/products/flockofbirds.php

${ }^{2}$ http ://www.mpb - technologies.ca/space/freedom $6{ }_{2} 000 /$ f6s / freedom 6 s.html

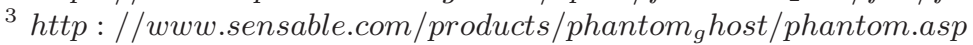

${ }^{4}$ http : //www.computermotion.com/products/index.html
} 


\section{References}

1. Debbins, J.P. and Riederer, S.J. and Rossman, P.J. et al., Cardiac Magnetic Resonance Fluoroscopy, Magnetic Resonance in Medicine, 36 (1996) 588-595

2. Gardstrom, 3D Navigation for real-time MRI using six degrees of freedom interactive devices, Master thesis. Department of Science and Technology, Linkoping University, SE-601M. Sweden, 2003.

3. Kerr, A.B. and Pauly, J.M. and Hu, B.S. et al., Real-Time Interactive MRI on a Conventional Scanner, Magnetic Resonance in Medicine, 38 (1997) 355-367

4. Hardy, C. J.and Darrow, R. D. and Pauly, et al., Interactive Coronary MRI. Magnetic Resonance in Medicine. 40, (1998) 105-111

5. Hayward, V. and Gregorio, P. and Astley, O. et al., Freedom-7: A High Fidelity Seven Axis Haptic Device With Application To Surgical Training. In Experimental Robotics V, Casals, A., de Almeida, A. T. (eds.), Lecture Notes in Control and Information Science 232, pp. 445-456, 1998.

6. Radau, P. and Hu, N. and Stainsby, J. and Wright, G.A., Visualization System for Real-time Scan Plane and Catheter Navigation. Accepted to 12th Scientific Meeting and Exhibition of the International Society for Magnetic Resonance in Medicine, 2004.

7. Salisbury, Jr. et al., Master having redundant degrees of freedom, US Patent \#6,714,839 (2004).

8. Stainsby, J.A and Hu, N. and Yi, D. and Radau, P and Santos, J.M. and Wright, G.A., Integrated Real-Time MRI User-Interface 12th Scientific Meeting and Exhibition of the International Society for Magnetic Resonance in Medicine, PP. 120. May 5-21, 2004, Japan.

9. Sussman, M.S. and Stainsby, J. A. and Robert, N. and Merchant,N. and Wright, G.A., Variable-Density Adaptive Imaging for High-Resolution Coronary Artery MRI. Magnetic Resonance in Medicine, 2002, 48(5):753-764.

10. Yi, D., Computer Aided Display of 3D Angiograms, Using Graphics and Haptics, Ph.D. dissertation, ECE department, McGill University, Montreal, Canada (2002). 